\title{
Power Line Communication (PLC)
}

\author{
Eng. Hussa Allhaib
}

\begin{abstract}
Wide range of PLC technologies are used in multiple applications, varying from simple internet access to complex home automation. This paper represents an overview of installing, evaluating and testing the PLC adapters in a small environment, which consist of three story house and basement using a DevalodLAN power line adapter. In addition, measuring the speed of the PLAs in different floors and in different distances away from the router.
\end{abstract}

\section{INTRODUCTION}

Power-line communication (PLC) is a method used to connect devices either to other devices or to the internet using existing electrical wires in a building, in which the wires carry both AC electric power transmission and data simultaneously. It can be a means of expanding an existing network into other new places without new wiring, simply by utilizing every power socket as internet port using PLC adapter in addition to the ability of transforming electrical socket to $\mathrm{Wi}-\mathrm{Fi}$ hot spot.

In other words, HomePlug standards and technology use the existing electrical wires in a building as network cables to communicate data between PLC's adapters by extending the existing network in order to provide wired and wireless connectivity from every electrical outlet. For example, a computer and a printer are placed in different locations in an area not covered by existing Local Area Network (LAN). Thus, the ability to connect to the internet is possible by using the existing electrical wires and three PLC adapters. These three PLC adapters are placed as follows: connecting one adapter to a router of an available wired Local Area Network (LAN) via its network port, the second adapter is to connect the computer, and the third one is to connect an Ethernet-ready device like a printer. When the three adapters are plugged into the wall sockets they will have a network connection via the electrical wiring in between the computer and the printer on one side and the router on the other side (connecting devices to the internet) and in between the computer and printer (connecting devices to one another). Some networking devices, such as switches or routers have built in power line connectivity, which will enable them to access the internet ones plugged into the power socket.

\section{METHODOLOGY}

Power-line setup connection can be extended as large as the building they are used in; its solution starts with minimum of two adapters. Plugging in The first adapter plugs into a power outlet then connects it the router. Whereas the second adapter is plugged in another power outlet near the internet enabled device you want connected or in a spot that the Wi-Fi signals cannot reach. The first adapter takes the Ethernet protocol used by your router and converts it into a Powerline protocol that uses electrical signals to send data through the wiring in the house to the second device. The second adapter receives the Powerline protocol and returns it back into an Ethernet signal.

The Technology of the Power Line Adapter (PLA) uses standards called HomePlug to allow communication between any two adapters to ensure the transmission of data over electrical wires with no corruption and noise. Which mean that Power-line networking can provide both wired and $\mathrm{Wi}-\mathrm{Fi}$ connection to any device with Ethernet port or Wi-Fi enabled can connect to the internet. The technology and standards of HomePlug first introduced in 2001 as HomePlug 1.0 since then upgrading took place and produce enhanced protocols. Currently the best Powerline protocol in the market is HomePlugAV2, because of its flexibility in contrast with the older HomePlug standard, it is designed to increase speed, extend network coverage, reduces power consumption by providing sleeping mode and more secure

\section{Evaluation}

The powerline network has been tested in three story house with a basement were the main router with DSL line located in the ground floor. Each floor including the basement had a PLA plugged and connected directly to the router via Ethernet cable. Now, every power socket in the house floor can act as Ethernet port or Wi-Fi adapter using the proper PLA as in fig (1). 


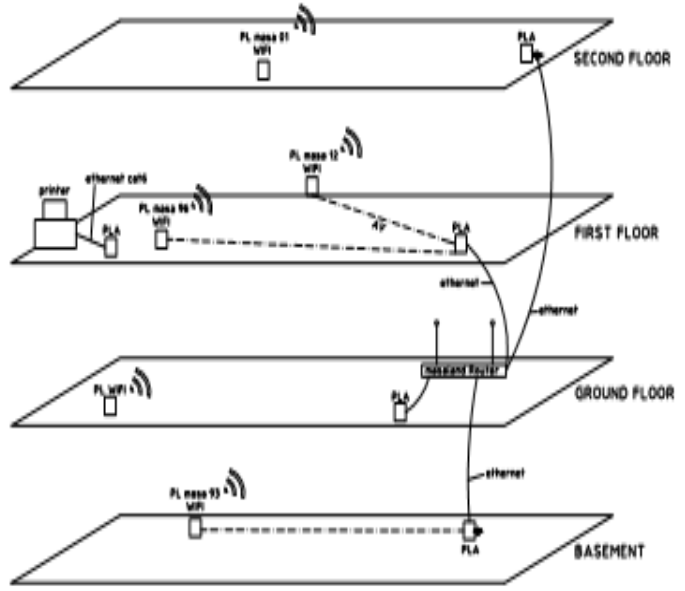

\section{- ethernet}

$=-1-A V / 2$

PLA power line Adapter

S

Fig (1)

In a study to evaluate the speed of data transmission against location of $\mathrm{Wi}-\mathrm{Fi}$ adapter (distance) result was collected and tabulated as shown in table (1).

\begin{tabular}{|c|c|c|c|c|c|}
\hline & \multicolumn{5}{|c|}{ adapter (masa 12) - first floor } \\
\hline $\begin{array}{c}\text { Distance } \\
\text { away from } \\
\text { PLA (meter) }\end{array}$ & $20.0 \mathrm{~m}$ & $25.0 \mathrm{~m}$ & $28.0 \mathrm{~m}$ & $32.0 \mathrm{~m}$ & $35.0 \mathrm{~m}$ \\
\hline $\begin{array}{c}\text { Down load } \\
\text { speed (Mbps) }\end{array}$ & 8.1 & 8.9 & 8.2 & 7.3 & 7.3 \\
\hline
\end{tabular}

Table (1)

\section{the distance from the PLA adapter VS the speed of the internet}
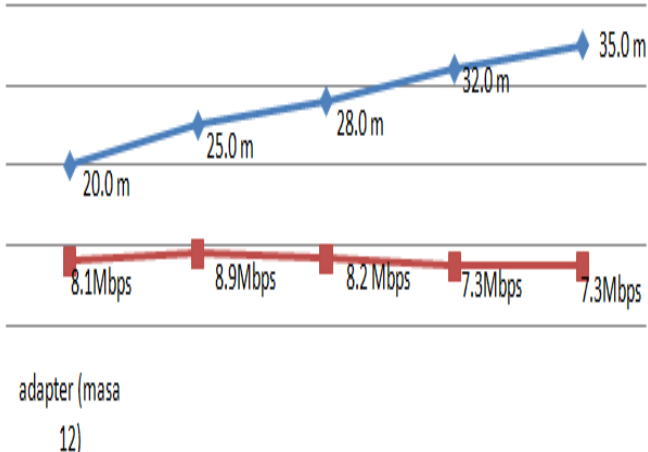

12)

\section{+ distance (meter) $\quad-$ speed (Mbos)}

Minor distance changes did not affect the speed of data download because the speed of the DSL adapter is very slow in contrast with the distance. On the other hand, download speed is affected when more than one device is connected to the internet; then the speed is divided between the devices.

\section{Findings}

- The three different electrical phases (yellow, blue, red) has no effect on the adapter communication (no interference).

- Regardless of the location of PLAs, the speed received from the devices is fully consistent with the ISP speed. There is no interference of data transmission and speed due to distance.

- Reliable in data transmission and speed as well as dependable.

\section{Security and Privacy}

Electrical signals can be hacked, just as eavesdropping on a $\mathrm{Wi}-\mathrm{Fi}$ signal is possible. Therefore, it is important to pick a Powerline adapters with the best encryption technology available (WPA, wpa2). Adapters usually come with security buttons that, when activated, encrypt communications.

\section{Advantages and disadvantages:}

\section{i. Advantages:}

- Installation of Additional cables and network hardware is not required to set up a home networking: The PLC uses the existing electrical network for communication.

- Easy setup: Powerline networking is easy to install; a person can do it in just a few minutes. If there is only one or two devices in your home that need the Internet, Powerline may be the most consumer-friendly solution for you.

- The availability of communication service can be on every outlet: The PLC uses the existing power outlets and powerlines in the house to send and receive information's from one device to another.

- Saving money on installations: The cost of installation is lower than other communication system, suppose you have a device that can connect to the Internet with a wired Ethernet connection, but it doesn't have Wi-Fi. Unfortunately, your router is on the other side of the house therefore you need to connect wires, which are inconvenient in our case. Purchasing a pair of Powerline adapters is a faster and more affordable solution.

- Solving Wi-Fi difficulties: There are some places that Wi-Fi can't be reached. Heavy interference or extra-large houses may make it too difficult to use a wireless connection. In these cases, Powerline adapters can supplement Wi-Fi networks or help provide one-on-one solutions for devices that really need a wired connection. This may also help with other problems, like 
spotty streaming or slow speeds that you would like to improve.

\section{ii. Disadvantages:}

- A common technical problem between wireless and power-line communication, particularly those of wide-bandwidth radio signals operating in a streamed environment. Radio interference, for instance, is a concern of radio groups.

- The efficiency and quality of the PLA connection are depending on the local electrical system quality. Meaning that improper wire installation and connection (e.g. circuit breakers) between the cables leads to bad performance causing connection disturbance.

- Power line adapters needs to update the firmware.

\section{REFRENCES}

[1]. Mannan, Abdul, D. K. Saxena, and MahrooshBanday. "A Study on Power Line Communication." International Journal of Scientific and Research Publications 4.7 (July 2014): n. page. Web. <http://www.ijsrp.org/research-paper0714/ijsrp-p3152.pdf $>$.

[2]. Akarte, Vivek, NitenPunse, and AnkushDhanorkar. "Power Line Communication Systems." International Journal Of Innovative Research In Electrical, Electronics, Instrumentation And Control Engineering 2.1 (January 2014): n. pag. Web.

[3]. <http://ijireeice.com/upload/2014/january/IJ IREEICE4A_a_vivek_powerline_communic ation.pdf $>$.

[4]. Lacoma, Tyler. "Is Wi-Fi Too Unreliable? Powerline Networking May Be What You Need." Digital Trends. N.p., 08 May 2016. Web. $28 \quad$ Mar. 2017. <http://www.digitaltrends.com/computing/e verything-you-need-to-know-aboutpowerline-networking/>.

[5]. Murty, Rohan, JitendraPadhye, Ranveer Chandra, Atanu Roy Chowdhury, and Matt Welsh. "Characterizing the End-to-End Performance of Indoor Powerline Networks." (n.d.): n. pag. School of Engineering and Applied Sciences, Harvard University. Web. 28 Mar. 2017. $<$ http://www.eecs.harvard.edu/ rohan/paper s/powerline-tr.pdf $>$.

[6]. http://en.wikipedia.org/wiki/Power_line_co mmunication . 